\title{
Probing Mobile-point-defect-mediated Nanodomain Evolutions in Ferroelastic- ferroelectrics Under High Stress with In-situ TEM
}

Yu Deng ${ }^{1}$, Jim Ciston ${ }^{2}$, Karen Bustillo ${ }^{3}$, Colin Ophus ${ }^{2}$, Ruopeng Zhang ${ }^{4}$, Chengyu Song ${ }^{2}$, Christoph Gammer $^{5}$ and Andrew Minor ${ }^{2,4}$

${ }^{1}$ Nanjing University, Nanjing, Jiangsu, China (People's Republic), ${ }^{2}$ Lawrence Berkeley National Laboratory, Berkeley, California, United States, ${ }^{3}$ NCEM, Molecular Foundry, LBNL, Berkeley, California, United States, ${ }^{4}$ University of California-Berkeley, Berkeley, California, United States, ${ }^{5}$ Austrian Academy of Sciences, Leoben, Wien, Austria

Ferroelastic-ferroelectrics are multi-functional materials with attractive applications such as actuator, memory devices and flexible/wearable electronic devices [1-7]. This class of intrinsically brittle materials exhibits unique unconventional deformation mechanisms that could be potentially utilized to engineer novel electric-mechanical components. Notably, the close-correlated domain evolutions and phase transformations in ferroelastic-ferroelectrics is reported to generate a complex hierarchical structure that is responsible to the superelastic deformation behaviors of the materials at nanoscale [7]. By applying high stress to the material, hierarchical nanodomain evolutions can be introduced into ferroelasticferroelectrics, effectively tuning their properties. However, the complex nanodomain evolutions are challenging to understand: the domain mobility, the distributions of local strains and mobile point defects at domain walls, and the growth of the bundle domain structures have been discussed for a long time with controversy [1,2]. Small scale mechanical in-situ TEM observations provide unique real-time capability of capturing the nanodomain evolution while the stress field is applied. Here, by applying in situ TEM mechanical tests couple with 4D-STEM techniques that are capable of generating nm-resolved strain mapping in an aberration-corrected transmission electron microscope [1,4,5,6], we studied free-standing single-crystal $\mathrm{BaTiO}_{3}$ and PMN-PT sub-micrometer pillars, to show the mechanism of the mobile-pointdefect-mediated nanodomain evolutions (Figure 1) in ferroelastic-ferroelectrics under high stress. This reversible domain-mediated deformation mechanism allows for superelastic deformation of nominally rigid single crystal oxide nanowires. The use of 4D-STEM to dynamically map the ferroelectric domain structure and local strain state over large fields of view is transformative to our ability to understand this complex deformation behavior in a quantitative manner.

\section{Acknowledgements}

The authors acknowledge support by the National Natural Science Foundation of China (Grants Nos. 50802039) and Natural Science Foundation of Jiangsu Province, China (Grant Nos. BK20151382), and the National Science Foundation through the STROBE Science and Technology Center. RZ acknowledges funding from the US Office of Naval Research under Grant No. N00014-12-1-0413 and N00014-17-12283. The electron microscopy work was performed at the Molecular Foundry, Lawrence Berkeley National Laboratory, which is supported by the U.S. Dept. of Energy under Contract \# DE-AC0205CH11231. CO acknowledges additional support from the U.S. Department of Energy Early Career Research Program. HGB and JC acknowledge additional support from the Presidential Early Career Award for Scientists and Engineers (PECASE) through the U.S. Department of Energy. 


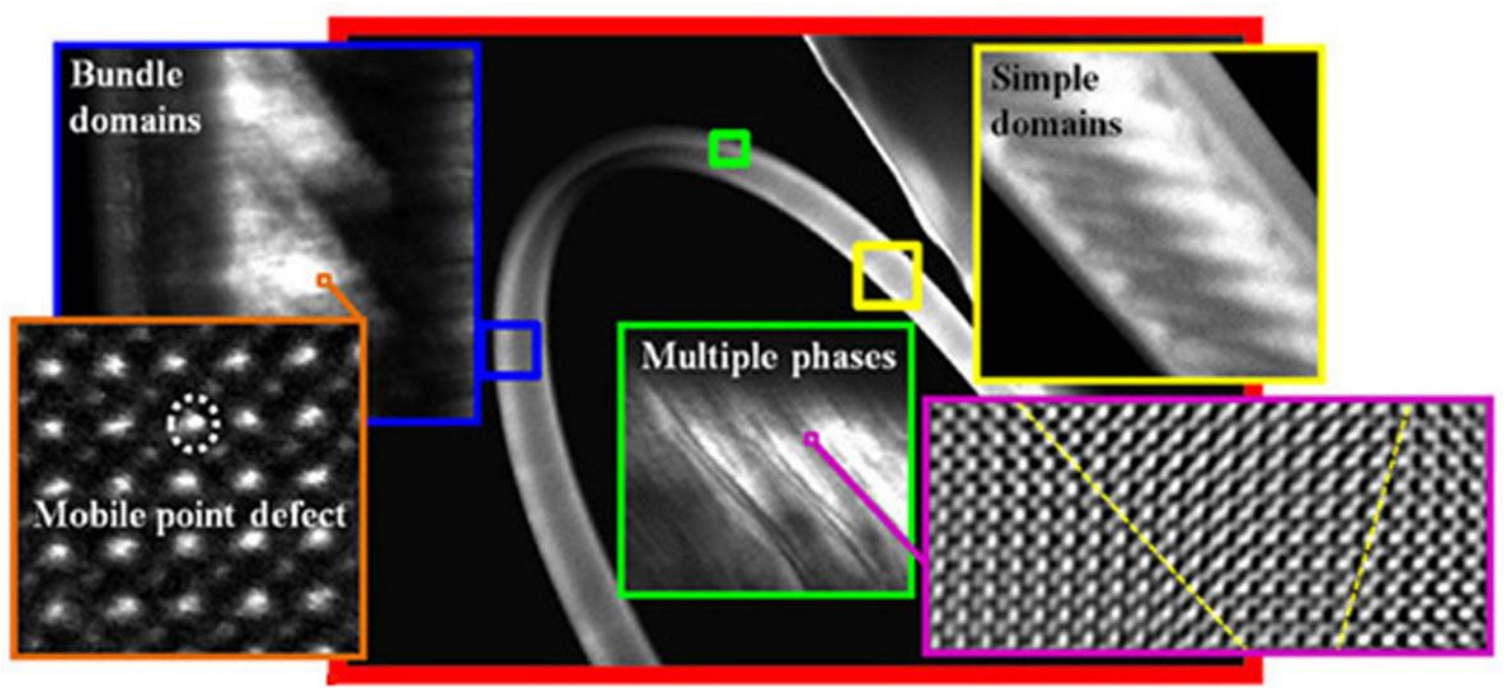

Figure 1. The mobile-point-defect-mediated nanodomain evolutions in a free-standing single-crystal BaTiO3 sub-micrometer pillar under heavily bending.

\section{References}

1. Y. Deng, C. Gammer, J. Ciston, P. Ercius, C. Ophus, K. Bustillo , C. Song, R. Zhang, D. Wu, Y. Du, Z. Chen, H. Dong, A. G. Khachaturyan, A. M. Minor, Acta Materialia 181, 501 (2019).

2. G. Catalan, J. Seidel, R. Ramesh, J. F. Scott, Modern Phys. 84, 119 (2012).

3. Y. L. Tang, Y. L. Zhu,X. L. Ma,S. J. Pennycook, et. al. Science 348, 547 (2015).

4. C. Gammer, V. B. Ozdol, C.H. Liebscher, and A.M. Minor, Ultramicroscopy, 155, 1 (2015).

5. V.B. Ozdol, C. Gammer, X.G. Jin, P. Ercius, C. Ophus, J. Ciston, A.M. Minor, Applied Physics Letters, 106, 253107 (2015).

6. Deng, Yu, et al., Advanced Materials, 1906105 (2019).

7. Zhang, J. X., et al., Nature nanotechnology, 6,97 (2011). 